\title{
REPLI-g Pooled DNA
}

National Cancer Institute

\section{Source}

National Cancer Institute. REPLI-g Pooled DNA. NCI Thesaurus. Code C156437.

A genomic DNA sample produced from a pool of samples from several subjects that is amplified using REPLI-g technology. 